\section{$\underset{\text { \& migrations }}{\text { hommes }}$}

\section{Hommes \& migrations}

Revue française de référence sur les dynamiques

migratoires

1315 | 2016

Ondes de choc

\title{
La diaspora morisque : une histoire globale méconnue
}

\section{Marc Terrisse}

\section{(2) OpenEdition \\ Journals}

\section{Édition électronique}

URL : http://journals.openedition.org/hommesmigrations/3737

DOI : 10.4000/hommesmigrations.3737

ISSN : 2262-3353

\section{Éditeur}

Musée national de l'histoire de l'immigration

\section{Édition imprimée}

Date de publication : 1 juillet 2016

Pagination : 124-129

ISBN : 978-2-919040-36-0

ISSN : $1142-852 X$

\section{Référence électronique}

Marc Terrisse, "La diaspora morisque : une histoire globale méconnue », Hommes \& migrations [En ligne], 1315 | 2016, mis en ligne le 02 janvier 2017, consulté le 15 septembre 2020. URL : http:// journals.openedition.org/hommesmigrations/3737 


\section{LA DIASPORA MORISQUE : UNE HISTOIRE GLOBALE MÉCONNUE}

Par MARC TERRISSE, historien, chercheur associé au CNRS, laboratoire Cerhio (Le Mans).

es Morisques sont des musulmans espagnols

Lqui ont été contraints de se convertir au catholicisme dès 1502. Cette date marque la mise en place des édits de conversion qui obligent les musulmans à embrasser la foi catholique. Ces édits abrogent la signature d'accords qui leur permettaient de conserver leurs pratiques et leurs coutumes à la suite de la Reconquista, marquée par la prise de Grenade en 1492. Persécutés par le système de l'Inquisition, ils sont expulsés par le roi Philippe III entre 1609 et 1612 à la suite de plusieurs révoltes.

Les Morisques apparaissent comme les héritiers de la culture arabo-andalouse qui s'est développée dans toute la péninsule ibérique depuis la première moitié du VIII siècle. Ils forment d'importantes communautés dans la région de Valence, l'Aragon, la Castille et l'Andalousie. Leur expulsion a eu des répercussions non seulement sur la France de l'époque, mais également sur l'Empire ottoman ou encore le Maroc. Les rébellions, tout comme les expulsions de Morisques, sont appréhendées en fonction des spécificités régionales par les spécialistes espagnols. Pour ce qui est de la France, les vagues de migration et d'installation sont étudiées au niveau local. Le Maroc, la Tunisie et l'Algérie relatent, de leur côté, la diaspora à travers l'histoire urbaine de Rabat, Tétouan, Tanger, Alger ou encore Tunis. L'his- toire des Morisques revêt enfin une dimension contemporaine en raison d'enjeux de mémoires convoqués par la politique.

\section{Les Morisques des différentes régions espagnoles}

Des témoignages relatifs au maintien de ces musulmans espagnols convertis sont visibles dans plusieurs régions, tandis que des sources qui relatent des révoltes, signes avant coureurs de leur expulsion, nous sont parvenues. Ces sources proviennent en premier lieu de l'architecture. Nombre de bâtiments d'époques morisques comme des églises, des maisons ont traversé les siècles dans plusieurs régions d'Espagne. Les sources écrites proviennent des différents textes promulgués par le pouvoir royal concernant les conversions forcées, puis les expulsions, mais également des registres de baptême et de mariage. Ces textes ont été étudiés par la recherche pour mieux appréhender l'histoire des Morisques. Des sources iconographiques, essentiellement des tableaux illustrant l'expulsion des Morisques, viennent compléter cet ensemble.

Parmi les nombreuses communautés morisques (ce sont des communautés territoriales distinctes) qui subsistent en Andalousie malgré les 
conversions forcées, celle de Grenade, regroupée dans le quartier de l'Albaicin, illustre l'histoire de ces populations tout au long du XVIe siècle. Les Morisques restent majoritaires dans le quartier jusqu'à leur expulsion en 1571, précédée, en 1568, par une révolte écrasée rapidement. Les travaux de Bernard Vincent' nous offrent la possibilité de mieux comprendre l'histoire de ce quartier.

Des maisons patriciennes habitées par des grandes familles morisques nous sont parvenues. Elles témoignent de l'importance de cette communauté après la fin du processus de Reconquista et avant leur expulsion. La calle (rue) Horno del Oro, située au cœur du quartier de l'Albaicin renferme ainsi un certain nombre de maisons morisques relativement bien conservées par rapport à leur aspect originel. Située au numéro 14, la Casa morisca de la calle Horno del Oro est classée monument national depuis 1922. L'étage supérieur date du XVIe siècle. Sa partie centrale reprend les codes de l'architecture mudéjar (plafond à caissons décoré de peintures reprenant des symboles islamiques comme l'étoile octogonale). Toujours dans l'Albaicin, l'ensemble dit de la Casa del Chapiz se compose de deux maisons datant du XVIe siècle. Elles ont appartenu aux Morisques Hernán López el Feri et Lorenzo el Chapiz. Ces deux personnages représentaient en quelque sorte les grandes familles morisques du quartier, membres de l'élite sociale et culturelle encore présente malgré l'Inquisition et la volonté de mettre à mal l'identité morisque. L'opulence que dégagent les édifices illustre le rang social et économique qu'occupaient ses propriétaires au sein du quartier et de la ville.

Située non loin de l'Andalousie, Hornachos, petite cité de l'Estrémadure, renferme une histoire singulière marquée par les Morisques. Même après la Reconquête chrétienne intervenue au XIII' siècle, les musulmans n'ont jamais réellement formé une minorité mais plutôt une majorité, officielle- ment jusqu'aux édits de 1502. Ils occupent toutes les fonctions au conseil de la ville face à une minorité de chrétiens parmi lesquels on trouve le gouverneur ou encore un ecclésiastique. En 1502, tout comme lors de la reconquête de la ville par la couronne castillane, les musulmans acceptent la conversion et restent à Hornachos². Mais c'était sans compter sur le processus d'Inquisition mis en place par les autorités de l'époque à l'encontre des Morisques, appelés désormais "nouveaux chrétiens ». Contraints à l'exil à la suite des expulsions de 1610, une bonne partie des Hornacheros quittent leur région pour la kasbah des Oudaïas de Rabat au Maroc. D'autres communautés morisques issues d'autres contrées ibériques se sont également établies dans la ville de Salé voisine de Rabat. Cette présence morisque suscite une émulation entre les diverses communautés qui sont, pour certaines, très impliquées dans la piraterie. Les Morisques d'Hornachos sont pour finir à l'origine de la République des Deux Rives formée par Rabat et Salés.

En Aragon, l'art mudéjar demeure un trait caractéristique. Cet art se distingue par la reprise de techniques et de décorations provenant de l'art islamique (arcs outrepassés, clochers reprenant les codes stylistiques des minarets, azulejos, marqueteries). La décadence puis l'extinction de celui-ci aux XVIe et XVIle siècles est marquée par l'expulsion des Morisques aragonais en 16091610. Cette période a laissé des exemples architecturaux remarquables visibles dans différentes églises à Saragosse (San Pablo). Les tours clochers, directement inspirées des minarets à Muniesa, Mara, Tierga, Alcubierre, Ricla, Urrea de Jalon, Villamayor ou encore l'église Notre-Dame-de-l'Assomption décorée d'azulejos d'Utebo, illustrent cette importance de l'art mudéjar tardif. Les conversions forcées des Morisques débutent à partir de 1526 en Aragon. Elles sont retardées par rapport à la Castille qui connaît ce mouve- 


\section{REPÉRAGE}

ment dès 1502. En effet, la noblesse s'appuyait sur la population morisque pour ce qui concernait la possession des terres et elle fit pression pour retarder les conversions forcées. Une fois le processus lancé pour convertir les populations musulmanes, il devint nécessaire de construire de nouvelles églises pour permettre aux nouveaux chrétiens de pratiquer leur culte. L'art morisque se développe donc en premier lieu dans ce cadre.

\section{L'étude des sources iconographiques}

Les sept tableaux décrivant l'expulsion des Morisques depuis Valence en 1609 et peints entre 1612 et 1613 font office de source iconographique majeure. Valence et sa région ont joué un rôle fondamental dans l'histoire de la diaspora. L'originalité de ces œuvres réside dans le fait qu'elles ont été peintes peu après l'expulsion et qu'elles semblent saisir sur le vif cet événement historique.

Ces tableaux retracent les principales étapes de l'expulsion : l'embarquement des Morisques, la révolte et l'arrivée à Oran marquant le début de l'exil. C'est le Marquis de Caracena qui passa la commande de ces peintures sur ordre du roi Philippe III d'Espagne, grand commanditaire de ces œuvres. Les peintres, longtemps méconnus, ont été identifiés dans le cadre de la première exposition publique liée aux tableaux et grâce à des recherches d'envergure. Ces peintres sont originaires de la région de Valence et ont probablement vécu, voire assisté à l'expulsion. Ils n'ont certes pas une envergure nationale ou internationale, à l'image d'un Velázquez, mais possèdent une réputation régionale bien enracinée.

Pere Oromig, Embarquement des Morisques au Grao de Valence (1612-1613), (L. 1,10 × 1,74, huile) Ce tableau décrit l'embarquement des Morisques dans le Grao, quartier qui n'existe plus aujourd'hui et qui se situait à proximité du port de la ville de Valence. II peut être divisé en trois parties. À gauche, les Morisques patientent sur les quais devant des bateaux, avant de quitter le pays qui les a vus vivre durant neuf siècles. Ils sont de petite taille par rapport au reste du tableau. Le peintre met en relief l'intensité dramatique du départ. Au centre, le port avec une croix symbolisant l'importance de la foi catholique triomphante. On trouve également les protagonistes ayant décidé l'expulsion, l'élite locale mise en valeur par sa grande taille. On dénote ici une forme de propagande. À droite, on aperçoit les murailles fortifiées du quartier avec les spectateurs de l'expulsion.

Jerónimo de Espinosa, Rébellion des Morisques de la Sierra de Laguar (1612-1613), (L. 1,09 × 1,73, huile) Le tableau intitulé Rébellion des Morisques de la Sierra de Laguar représente la principale réaction violente des Morisques contre l'expulsion. Les soldats espagnols sont majoritairement représentés sur le tableau tandis que les rebelles morisques demeurent repliés sur le plateau en haut de la colline. La force de la chrétienté est ici mise en valeur devant des Morisques désordonnés, représentés de façon beaucoup plus petite que les forces royales.

Vicente Mestre, Débarquement des Morisques dans le port d'Oran (1613), (L. 1,10 × 1,73, huile) Le Débarquement des Morisques dans le port d'Oran est le dernier tableau de la série. Oran reste à l'époque occupée par les Espagnols tandis que la région est placée sous le joug ottoman. Les populations locales accueillent violemment les Morisques car ils les considèrent comme des envahisseurs espagnols. Les survivants parviennent malgré tout à s'implanter progressivement dans les villes voisines où leur intégration prend un certain temps. Considérés comme des musulmans en Espagne, comme des Espagnols 
en terre d'islam, beaucoup sont islamisés de nouveau. La plupart des Morisques s'établissent au Maroc, en Algérie, en Tunisie ou dans d'autres régions de l'Empire ottoman. II y a eu cependant des retours en Espagne, difficilement quantifiables mais sans doute peu nombreux ${ }^{4}$.

\section{Les Morisques en France}

Les Morisques quittaient déjà l'Espagne avant les décrets d'expulsions. Des manuscrits relatent leurs itinéraires passant par la France et conseillent aux Morisques de franchir les Pyrénées en se faisant passer pour des pèlerins. Alonso Lopez fait partie de ces individus. Appartenant à l'élite morisque aragonaise, il est à la fois homme d'affaires et espion pour la cour de France avant de devenir le proche conseiller de Richelieu ${ }^{5}$. Le Béarn, le Pays Basque, la région bordelaise, le Languedoc et la Provence accueillent la majorité des Morisques expulsés, qui arrivent soit par la mer, soit en franchissant les Pyrénées. Les travaux de Louis Cardaillac sur le passage des Morisques s'appuient sur les archives de plusieurs villes, du Parlement de Paris et du ministère des Affaires étrangères. II utilise également les sources juridiques espagnoles. II dénombre 270000 Morisques expulsés d'Espagne pendant la période 1609-1612. En dehors des Morisques expulsés par la mer depuis Valence en 1610 et qui ont eu pour destination des régions musulmanes, les contingents issus des autres provinces espagnoles ont été en mesure de fouler le sol du Royaume de France ${ }^{6}$.
La Navarre française et le Béarn sont, à cette époque, un État protestant autonome avec le futur Henri IV à sa tête. Dedieu fait part d'échanges entre le parti protestant en France et les Morisques?. La rivalité entre les deux puissances française et espagnole trouve donc un terrain de déstabilisation mutuelle dans le cadre du soulèvement des minorités religieuses. Morisques aragonais et castillans restent les plus nombreux à tenter de traverser les Pyrénées. Si certains d'entre eux empruntent un itinéraire passant par le pays catalan et débouchant sur les grandes villes languedociennes, d'autres remontent vers la côte atlantique et stationnent à Bayonne, Saint-Jean-de-Luz, Bordeaux et même au-delà. Une certaine Marion Manuel, domestique à Paris, est ainsi mentionnée dans le Mercure François de 1610 en raison de son hermaphrodisme. Une source montre qu'une cinquantaine de familles se fixent dans la région de Bayonne, peut être de façon provisoire ou définitive. Ce chiffre se base sur une lettre provenant d'un Morisque installé à Saint-Jean-de-Luz en 1611. Louis Cardaillac assure en outre que des potiers et un maréchal-ferrant morisques ont choisi de s'installer à Biarritz après l'expulsion de 1609 car ils répondaient à une pénurie locale.

Des expulsions se font par bateaux depuis l'Espagne avec pour destination les ports provençaux comme Marseille, La Ciotat ou encore Fréjus. Dans les sources écrites provençales, on retrouve le nom de morisques, moriscous, grenadins, granatins, en particulier dans les registres paroissiaux mentionnant les baptêmes et les décès ${ }^{8}$. C'est cette clef de lecture qui permet de retrouver

\footnotetext{
4. Youssef El Alaoui, "L'expulsion des Morisques, l'heure fatale à travers les tableaux de la collection Bancaja ", in Jean-Claude Arnould (dir.), L'Instant fatal. Actes du colloque international organisé par le CÉRÉDI et le GEMAS (Université de la Manouba, Tunis), les jeudi 13 et vendredi 14 décembre 2007, 2009. 5. Youssef El Alaoui, "Alonso López. Itinéraire d'un Morisque espagnol au service de la France de Richelieu ", in Les représentations de l'individu et de la société : rapports et conflits, projets de la société du futur par rapport à l'individu. Évolutions-involutions, progression-régression, colloque à l'université de Rouen, les 13, 14 et 15 février 2008. 6. Louis Cardaillac, "Le passage des Morisques en Languedoc ", in Annales du Midi, Revue archéologique, historique et philologique de la France méridionale, vol. 83, n 103, 1971. 7. Jean-Pierre Dedieu, "Entre religión y política: los moriscos ", in Manuscrits, $n^{\circ}$ 12, 1994, pp. 63-78. 8. Pierre Santoni, "Le passage des Morisques en Provence (1610-1613)", in Provence historique, vol. 185, n 46, 1996.
} 


\section{REPÉRAGE}

leurs traces dans les archives. Certains ont essayé de s'implanter en Provence, sans doute de façon provisoire, en exerçant des métiers à connotation artisanale. Les travaux de Bernard Romagnan ${ }^{9}$ mettent en exergue des délibérations municipales des années 1612-1613 qui montrent que les consuls de Toulon, de Sainte-Tulle ou encore de Gréoux-les-Bains avaient approuvé l'embauche d'artisans aragonais, donc probablement morisques, pour effectuer certaines tâches. l'historien cite par ailleurs les personnages de Maître Louis de Molina, charpentier, de Diego Perez, cordonnier, ou encore de Philipon de Caillas, teinturier, tous trois de Grenade, qui prirent possession à La Napoule d'une terre pour 18 livres. À Fréjus, on trouve trace d'un contrat concernant une Marie Mariplon, fille de Morisques, qui devient servante. Certains devinrent propriétaires comme Hiéronyme Mayou, maître maréchal aragonais qui achète une boutique à Hyères en 1616. Cette boutique fut louée pendant de nombreuses années à l'acheteur. Mayou y était donc installé depuis plusieurs années. Une présence modeste de Morisques se dessine ainsi dans le tissu local. Un certain nombre de marchands dits "Aragonais " semblent réaliser des affaires entre eux, s'appuyant sur un système d'entraide communautaire. Cavalères, Saint-Tropez ont également vraisemblablement vu s'installer quelques familles ou des individus. Les travaux de Bernard Romagnan identifient ainsi dans les actes de décès la présence d'un Mathieu en 1627. Mais d'autres cas ont été recensés dans des villes voisines.

Devant le flux croissant de migrants, la décision d'expulser les Morisques vers des régions de culture musulmane est prise sur ordonnance royale datant du 30 avril 1610. C'est le port d'Agde qui est choisi pour conduire les Morisques localisés en France vers les ports du Maghreb ou en direction du Proche-Orient. La présence des Morisques en Languedoc, après le rapatriement à Agde d'un grand nombre d'entre eux en terre d'islam, soit après les années 1610, perdure, à l'instar des villes de l'Ouest atlantique et en Provence. S'il reste bien difficile d'avancer un chiffre, des témoignages provenant de sources écrites montrent que ces derniers exerçaient des activités dans des cités languedociennes. Cardaillac parle ainsi, en 1611, d'artisans en peinture et marqueterie ayant embrassé la foi catholique et s'étant implanté à Béziers. Ce sont les plaintes des autres artisans dénonçant une nouvelle forme de concurrence qui permettent d'identifier leur présence. Les Morisques demandent en effet au duc de Ventadour de plaider leur cause auprès des autorités locales. Les correspondances du duc de Ventadour avec les consuls de la ville, conservées aux archives municipales de Béziers, témoignent de cette présence.

À Lunel, les registres de baptêmes de l'époque font la mention d'enfants dont les membres de la famille sont qualifiés de Grenadins, soit originaires de Grenade et donc morisques. On retrouve plusieurs Morisques à Montpellier et qui sont stigmatisés avec véhémence par la population et les autorités car ceux-ci sont encore qualifiés de musulmans et sont accusés de ne pas vouloir s'intégrer.

\section{Les enjeux contemporains liés à l'histoire des Morisques}

À l'occasion des 400 ans de l'expulsion de 1609 , l'Espagne a pris à bras-le-corps la compréhension historique de ces événements et a en parallèle développé un processus mémoriel ambitieux. Au-delà d'une actualisation et d'une valorisation 
d'un état des lieux de la recherche historique sur la question des Morisques, grâce à l'organisation de colloques, l'Espagne a valorisé le patrimoine et l'héritage légués par cette minorité. En outre, des documentaires pour le grand public ont été réalisés dans le cadre des événements dédiés à la commémoration de l'expulsion des Morisques. Des discours politiques ont accompagné l'ensemble des manifestations. Le Congrès des députés espagnols a voté une proposition visant à reconnaître l'injustice commise à l'égard des Morisques en 2009. Cette proposition a engendré un vaste débat dénonçant la porte ouverte laissée à d'autres minorités qui pourraient se saisir de ce processus de reconnaissance : les homosexuels, les esclaves africains ou encore les athées, les sorcières, etc.

Cette problématique mémorielle a connu en 2014 un nouveau rebondissement. L'Espagne a décidé d'accorder automatiquement la nationalité aux Juifs expulsés du Royaume en 1492. Cette initiative a ouvert la boîte de pandore sur la question des minorités religieuses chassées d'Espagne lors de I'Inquisition. Les descendants de Morisques ou se déclarant comme tels ont rapidement demandé le même traitement que les descendants de Juifs espagnols. Or l'Espagne reste très partagée sur l'éventualité d'un tel élargissement. Elle fait d'ailleurs preuve d'un certain embarras, car elle considère qu'une ouverture du droit à la nationalité espagnole pour les descendants de Morisques risquerait d'ouvrir les frontières de l'Union européenne à des pays en guerre comme la Tunisie, la Libye, la Syrie ou l'Irak. Compte tenu de la crise économique frappant le pays, elle ne se sent pas en capacité d'accueillir et d'intégrer ces migrants dont le nombre pourrait s'avérer relativement conséquent.

En ce qui concerne la mise en valeur du patrimoine et de l'histoire des Morisques, beaucoup de pays n'ont pas fait les efforts de mémoire de l'Espagne. On ne trouve ainsi que très peu de recherches et encore moins de vulgarisation de cette histoire au Portugal. Du côté de la France, peu de travaux rappellent la présence morisque. II n'y a ainsi aucun mémorial soulignant le rôle d'Agde comme centre de tri des nouveaux arrivants, la présence de réfugiés dans les ports atlantiques ou encore le rôle d'Alonso Lopez, conseiller de Richelieu, ayant vécu à Paris. Le rôle de Venise n'est guère plus mis en valeur puisque la ville, un peu à l'image d'Agde, a également servi de point de passage des expulsés, en particulier ceux qui souhaitaient gagner l'Empire ottoman et Istanbul.

Il faut aller du côté du Maghreb, de la Tunisie et du Maroc pour observer une mise en valeur de l'histoire et du patrimoine morisques. Des maisons, des quartiers entiers de villes comme Tunis, Rabat et Salé au Maroc sont identifiés pour avoir accueilli des Morisques. L'apport culturel, économique et social de ces populations est largement souligné auprès du grand public, en particulier à travers les programmes scolaires mais également par le biais de documentaires et de fictions réalisés pour la télévision. Toutefois, la mémoire collective a tendance à passer sous silence l'accueil relativement hostile réservé aux Morisques lors de leur arrivée en terre d'islam. 